\title{
Autism Associated with B-Vitamin Deficiency Linked to Sugar Intake and Alcohol Consumption
}

\author{
Raymond J. Shamberger
}

King James Medical Laboratory, 24700 Center Ridge Road, Westlake, Ohio 44145, USA

\begin{abstract}
Objectives: Autism rates in the United States are increasing at a rate of $10-15 \%$ per year. This study uses nutritional epidemiology and relates autism rates to the total B-vitamin intakes. The total amounts of B-vitamins are then compared to the previously established minimal daily requirements to see if the intakes are adequate. The apparent lower B-vitamins may result from the increased consumption of sugar and alcohol which are devoid of vitamins,
\end{abstract} minerals, protein, fat and antioxidants.

Study Design: The autism rate was then compared to the percent exclusive per cent breast feeding from 2000-2004 as well as 2007-2010. Other comparisons were made between the statewide exclusive breast feeding and the binge drinking per state. The percentage of infants who participated in Washington state WIC (Womens Infant \& Childrens) program were also compared to the autism rate in each county. The autism rate among 8 year olds when compared to the \% increase of sugar consumption from 2002 to 2010 also showed an increase in the autism rate.

Results: The total amounts of B vitamins in breast milk seemed to be inadequate compared to published mdrs. There was also a direct relationship to the autism rate with the women who were breast feeding from 2000-2004 and 20072010. Increased autism rates were related to increased sugar consumption and to an increased alcohol intake.

Conclusions: The mothers who are exclusively breast feeding should continue their prenatal vitamins. Sugar intake and alcohol consumption should be decreased. The results suggest that autism is nutritionally related.

Keywords: Autism, binge drinking, B vitamins, minimal daily requirements, WIC program, ADDM Network.

\section{INTRODUCTION}

The United States Department of Education reports autism is growing at a rate of $10 \%-15 \%$ annually. About 1.5 million children in general now have some form of autism with more than 500,000 cases under the age of 21. The total numbers include Classic autism, Aspergers Disorder, Retts Syndrome, Childhood Disintegrative Disorder and Pervasive Disorder (PDB). Boys have a 4 to 1 chance of developing autism than girls. Among the 4 million children born each year in the United States 24,000 of them will develop autism.

About 1 in 6 children had a developmental disability in 2006-2008 ranging from mild disabilities such as speech and language impairments to serious disabilities such as intellectual disabilities, cerebral palsy and autism. Autism increased $289.5 \%$, ADHD $33 \%$ while hearing loss decreased $30.9 \%$. Factors which make a child more likely to have an ASD includes both environmental and also genetic factors. Children who have a sibling with ASD occurs more often in a child with genetic fragile syndrome or tuberous sclerosis. Children with older parents are ar a greater risk of having an ASD. The critical period for developing ASD seems to be before, during and immediately after birth.

*Address correspondence to this author at the King James Medical Laboratory, 24700 Center Ridge Road, Westlake, Ohio 44145, USA; Tel: 440: 256-1223; Fax: 440: 835-2177; E-mail: Bobray@ameritech.net
Nutritional epidemiology can be very useful in linking nutritional processes to disease. Some historic examples can be found in the diseases of scurvy, berberi and meat consumption and colon cancer.

In 1947 Captain James Lind [1] on the HMS Salisbury were losing his sailors to a mysterious disease and suspected a dietary deficiency. He tested different diets with groups of 12 crew members. One group given oranges and lemons recovered from a mysterious disease later identified as scurvy.

There was also an unusually large occurrence of berberi among sailors who ate polished rice. This led Captain Tabaki to conclude that some dietary factors were responsible. Addition of milk and vegetables to the diet eliminated the disease. Williams [2] found it was caused by a lack of thiamine.

Sandhu [3] has done a systematic review of prospective cohort studies and linked meat consumption to colorectal cancer using a meta analytical approach.

Sometimes large amounts of time exist between the nutritional deficiency and the onset of the disease. This happens in childhood diseases such as autism and ADHD when the diseases appear after several years. Food diaries cannot be kept as in short term studies. However, exclusive breast feeding can be related to a disease in a population. In general, breast feeding 
rates and disease rates are recorded for several years. This makes it possible to relate disease incidence to exclusive breast feeding rates.

B-vitamin levels are known to be decreased by alcohol consumption. This may make it possible to compare alcohol consumption in the United States and also to South Korea whose autism rates are much higher than than the United States [4]. About $46 \%$ of the Korean men are heavy drinkers. In contrast, only about $3.5 \%$ of the United States are heavy drinkers (more than 6 drinks a day). The rate of alcohol consumption among Korean Americans is also highest among Asian emigrants to America.

This nutritional epidemiological article will explore several nutritional approaches to autism: vitamin levels of breast milk and bovine milk compared to the minimal daily requirements; the consumption of alcohol in the 50 states and its relationship to autism in those states; calculate additional WIC data in the counties of Washington; and compare the autism rates to WIC data in Washington and compare the "dry" and "wet" counties of Arkansas in regard to their autism rates. The Increasing sugar consumption and alcohol consumption will also be related to the increasing autism rate.

\section{MATERIAL AND METHODS}

Most of the original minimal daily requirements were established by the National Academy of Science by analyzing food intakes from several countries and then matching these amounts to nutritional deficiency disease in each country. Safe levels of each vitamin were determined and a margin of safety was added if a country was borderline. This study will compare the breast and bovine milk intakes calculated in this study to the minimal daily requirements. Calculations were made in the same way as the National Academy of Science study. The calculation was also similar to those found on labels of foodstuffs found at a food store.

To check if an average diet contains daily sufficient amounts of B vitamins, the milk drunk by an average infant in the United States in the first month (20 ounces/day) can be calculated. The average amount is then compared to the minimal daily requirements and to the average concentration in mother's milk according to the Department of Agriculture [5] Table 1. Thiamin and riboflavin were also compared in the same manner using data from the American Academy of Pediatrics [6]. These results were compared to the daily requirement of the infant. Regular bovine milk [5] was also included for comparison.

When the $B$ vitamins in mothers milk were compared to the minimal daily requirements (Table $\mathbf{1}$ ) they seemed to be low. Similar results were seen with data from the American Academy of Pediatrics. Both thiamine and riboflavin were inadequate. In contrast bovine milk seemed to be adequate in regard to the minimal daily requirements. The same pattern continued at 2 and 4 months between breast milk and bovine milk. Calculations were made in the same way as the National Academy of Science study. Calculations made were also similar to those found on the label of foodstuffs found at a food store.

The autism rate was compared to the WIC rate in the state of Washington in 2009. Participation represents $91 \%$ of eligible people for the program. To find the actual number of infants under 1 year, total participation is multiplied by 0.257 ( a national constant for several years). The number of participating infants in each county in 2009 was divided by the number of infants born that year for each county. Previously an

Table 1: The Total Daily Vitamin Content of 20 Ounces of Exclusive Breast Milk Feeding and Bovine Milk Daily for One Month and 30 Ounces of Milk in Month Four [1] Compared to the Minimal Daily Requirements (MDR)

\begin{tabular}{|c|c|c|c|c|c|}
\hline Vitamin & MDR/unit & Human-1 & Human-4 & Bovine1 & 0.27 \\
\hline \hline Thiamine & $0.2 \mathrm{mg}$ & 0.083 & 0.124 & 1.15 & 0.4 \\
\hline Riboflavin & $0.3 \mathrm{mg}$ & 0.21 & 0.32 & 0.6 & 0.9 \\
\hline Niacin & $2.0 \mathrm{mg}$ & 1.05 & 1.57 & 2.2 & 0.3 \\
\hline Pantoth. Acid & $2.0 \mathrm{mg}$ & 1.32 & 0.098 & 3.4 & 0.45 \\
\hline B-6 & $0.1 \mathrm{mg}$ & 0.065 & 44.6 & 2.6 & 53.5 \\
\hline Folate & $65 \mathrm{mcg}$ & 29.7 & 0.45 & 3.8 \\
\hline B-12 & $0.4 \mathrm{mcg}$ & 0.3 & & \\
\hline
\end{tabular}


Table 2: The Percent WIC Recipients in the Counties of Washington, New Jersey (3), Oregon (3) and the 50 States (3) Compared to the School Reported Autism Rates Per County

\begin{tabular}{|c|c|c|c|c|}
\hline State & Year & Counties & WIC/Autism & P \\
\hline \hline Washington & 2009 & 36 & -0.39 & $<0.02$ \\
\hline New Jersey & 2004 & 21 & -0.49 & $<0.02$ \\
\hline Oregon & 2010 & 30 & -0.39 & $<0.05$ \\
\hline All 50 states & 2004 & ---37 & $<0.02$ \\
\hline
\end{tabular}

inverse rate was also observed in New Jersey in 2004 and Oregon 2010 [7]. The results are summarized in Table 2 and are similar to those found in New Jersey and Oregon.

The WIC program helps low-income women to make nutritious choices. Food packages and food stamps are also provided. However, alcoholic beverages are not provided in the WIC program.

Alcohol consumption can cause certain alcohol related disorders such as fetal alcohol syndrome (FAS). FAS includes disorders ranging from minor abnormalities such as low birth weight to severe which includes mental retardation and facial abnormalities such as a short nose.

Mukherjee et al. [8] has also reported that drinking while pregnant can give babies FAS. He also compared autism and autistic traits in people exposed to heavy prenatal alcohol to a clinical series of 21 individuals in a nested case control study. The controls had not experienced prenatal alcohol exposure. The 21 with fetal alcohol spectrum disorders were assessed and $16(72 \%)$ met ICD-10 criteria for childhood autism.

In general, alcohol reduces the available B vitamins. Binge drinking may cause more of an extreme vitamin deficiency. The average binge drinking of 4 or more drinks among women 18-44 [9] was compared by Excel correlation to the autism rate for 8 year olds in that states (Figure 1). In addition, the total use of one or more drinks per month (not binged) was also compared by Excel correlation to the autism rate of 8 year olds in that state (Figure 1). The results showed a direct correlation of $r=0.36, P<0.05$. Comparisons of the total alcohol usage in the 50 states also showed a positive relationship of $r=0.419, P<0.01$. Older mothers who binge drank have a greater percentage of autistic children.

Binge drinking is very prevalent in South Korea which has a high rate of autism with a prevalence of $2.6 \%$ [6] In contrast, similar comparisons in Asia,

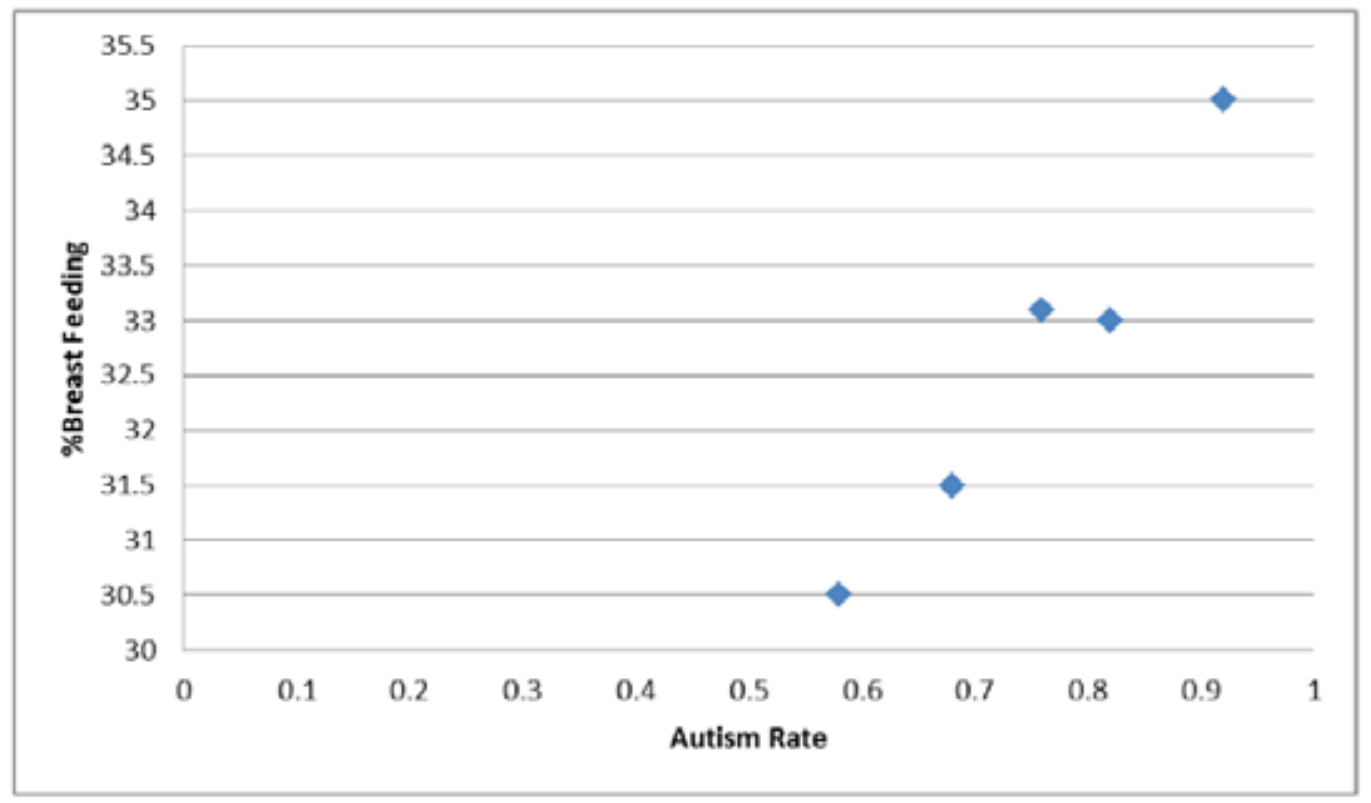

Figure 1: Comparison of the United States exclusive 3 month breast feeding [10] to the autism rate of 8 year olds (11) in the United States from 2007-2010. $R=0,979, P<0.001$. 
Europe and North America show a prevalence of About $1 \%$. The South Korean target population was 55,266 children ages 7-12. The greatest incidence of autism was among children from the general population rather than children from special education or disability registries. Their status was determined by an Autism Spectrum Screening Questionnaire. The study was assisted by Eric Fombonne, a professor of psychiatry at McGill University in Montreal.

Arkansas has several counties which are "dry " and "wet". Comparisons of autism rates were made between the 35 wet and 39 dry counties.

The historical increased sugar consumption from 1822 to 2005 was correlated to the increased autism in 8 year olds from 2002-2010. In 1822 the per capita sugar consumption was about 8 lbs per person per year and by 2006 reached over 100 lbs per year. Landen [11] constructed a graph which can be compared to the autism curve. $y=0.573 x-1041$ where $x=y e a r s$. Points can be calculated from the total national rate of autism from 2002-2010. Autism points were calculated from the total national autism rate according to the national ADDM Network (The Autism and Developmental Disabilities Network). This is a group of programs funded by CDC (Communicable Disease Center) to estimate the number of children with autism spectrum.

\section{RESULTS}

Because many of the B vitamins in this study have been found to be lower in breast milk, comparisons of thiamine, riboflavin, niacin, pantothenic acid, B-6 and folic acid seems to indicate they are below the minimal daily requirements. In contrast, regular bovine milk with the exception of niacin showed sufficient amounts.

Other possible nutritional links of the $B$ vitamins have been linked to autism. Lonsdale et al. [12] has done a pilot study on 10 autistic children showing the clinical and biochemical effects of thiamine tetrahydrofurfuryl-disulfide showing clinical improvements in eight of the children. Suren et al. [13] have found an association between maternal use of folic acid supplements and a reduction of not only the incidence of neural tube defects but also the risk of autism spectrum disorders in children. Of 93 children with autism spectrum disorders, $75 \%$ were found to have folic acid receptor antibodies in serum. Verbal communication, receptive and expressive language and behavior were improved in about one third of treated children. James et al. have observed cellular and mitochondrial glutathione redox imbalances in lymphoblastoid cells from children with autism [14]

Comparisons of the United States breast feeding per year [15] to the autism rate of 8 year olds for the years 2007-2010 showed a distinct relationship to autism [16] in the United States (Figure 2). A similar relationship has previously been observed for the years 2000-2004. The data suggests that some components of breast milk may be deficient in some nutrient. The Arkansas wet counties had a total autistic rate of 0.76 per 100 births and the dry counties had a rate of 0.63 per 100. Arkansas in general has a very high WIC

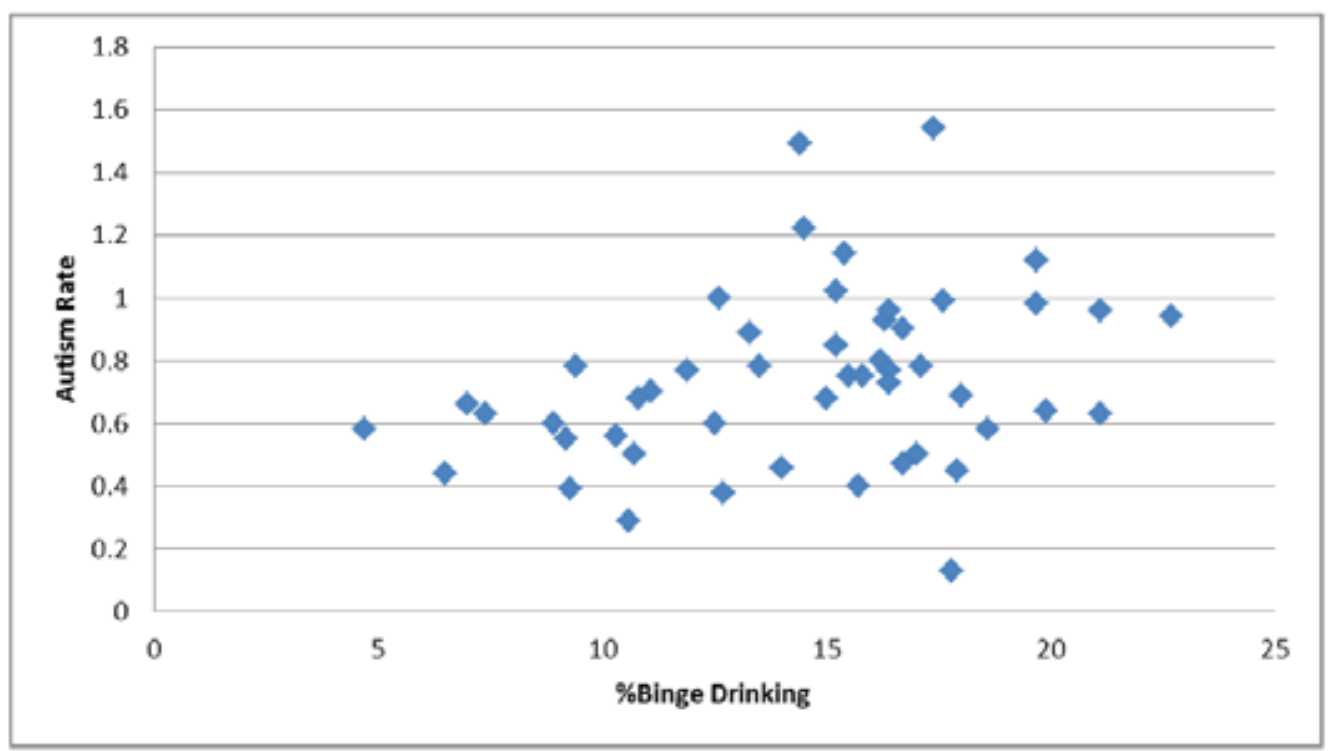

Figure 2: The average female age 18-44 binge drinking per state [5] compared to the autism rate of 8 year olds [11]\} in that state. $r=0.31, P<0.05$ 
Participation rate and also has a low autism rate. The rate difference does not seem to be significant but the overall results are consistent with an alcohol effect.

There was a direct significant relationship between the total National Autism Rate from the ADDM Network for the years 2002 to 2010 and the increasing American consumption of sugar.

\section{DISCUSSION}

Thiamine, riboflavin, niacin, pantothenic acid, B-6, folic acid and B-12 of breast milk seem to be below the minimal daily requirements. Most of the early MDR studies in various countries were done on foodstuffs and possibly whole milk. However, in this study we are comparing the established MDR on foodstuffs to an exclusive breast feeding diet and it is possible that the difference in the diets may be responsible for autism. The Amish children consume mostly bovine milk directly or indirectly. Robinson et al. [17] have reported an incidence rate of autism of only $0.28 \%$ in two Amish communities compared to the national rate of $1.09 \%$.

The two study correlations which were made from 2000-2004 and 2007-2010 both showed a direct relationship between autism percentage and the percentage of exclusive breast feeding. Both studies had a direct relationship of $\mathrm{P}<0.001$.

The South Koreans are ahead of the other ethnic groups in alcohol consumption and they have a very high rate of autism compared to the Americans. The tendency toward binge drinking among South Korean emigrants to the United States is about $24.6 \%$ and is two or three times greater than other Asian countries. In the urban areas of South Korea, families of children with developmental delays go to great lengths to avoid a diagnosis of chapae or autism which is a genetic mark of shame on the family by doctors. The diagnosis is a major obstacle to all of their children's chances of finding a suitable spouse. The mother accepts blame by accepting a reactive detachment disorder that was caused by extreme parental abuse or neglect.

In the Korean society it is understood that drinking is an essential part of networking which is necessary for success in the workplace. Many Koreans are first introduced to alcohol at universities and develop unhealthy drinking habits. In a poll of 4061 students by the Korean Alcohol Research Foundation in 2010, 71\% said they drank more than 5 shots of Soju in one sitting which is defined as binge by WHO (World Health Organization)
English women who binge drink during the second trimester are more likely to have an underweight baby or a small for gestational weight baby [18]. Growth restricted babies have a greater risk of having neonatal complications, including breathing problems, respiratory infections, hypothermia and impaired neurodevelopment.

Allen [19] has studied the relative importance of maternal status and intake and effects of B-vitamins on infant stature and function. The study estimated the percent intake of thiamin, riboflavin, vitamin B-6 and vitamin B-12, maternal supplementation and concentration in human breast milk and compared the recommended daily intake that infants could obtain from a deficient mother. Estimates were $60 \%$ for thiamine; $53 \%$ for riboflavin; $80 \%$ for vitamin B-6 and $16 \%$ for vitamin B-12. In a well nourished woman B vitamins are relatively stable about week 3 of lactation [20].

Excess alcohol intake decreases the absorption of folic acid, thiamine and riboflavin into the intestines Vitamin B-6 excretion is increased by alcohol and pantothenic acid is needed for the detoxification of alcohol. Extreme thiamine deficiency from alcohol can result in Warnike Korsakof syndrome. Riboflavin is needed for brain development. Flavin adenine nucleotide stimulates erythrocyte glutathione reductase and is known to prevent oxidative stress. B-12 injections are often given to alcoholics because the majority of the diet is replaced by alcohol. Adequate dietary levels of folic acid may help an alcoholic recover from a folic acid deficiency. B-6 is an important nutrient for normal behavioral development in infants [20] Breast fed babies have a lower level of B-6 than bottle fed babies. Higher B6 enables an infant to perform a learning task and has better control of autonomic nervous function such as breathing and temperature regulation.

While there are several B-vitamins that could be deficient and cause autism it is difficult to determine which ones are the most important. Thiamine and riboflavin deficiency seem to be the most likely.

Some of the factors such as siblings getting autism may not be answered by genetics. Siblings are sharing the same family food. Older mothers drink more alcohol more during pregnancy. The critical period for developing ASD seems to be before and immediately after birth. These latter factors give evidence that suggests autism is a may be caused by inadequate nutrition. 
In the United States the per capita sugar consumption is over $100 \mathrm{lbs}$ per year. The increasing sugar Intake shows a direct relationship to an increasing autism rate. Like alcohol excess sugar also needs B-vitamins for metabolism thereby reducing their availability. In medical practice when an alcoholic is brought into the emergency room they are administered an I.V. containing folic acid and thiamine instead of glucose which would cause additional brain damage.

There are several ways that a nursing mother can become B-vitamin deficient: a poor diet devoid of adequate B-vitamins due to bad dietary choices or poverty; consuming alcohol while nursing; and not having a sufficient intake of B-vitamins to cover the increased mdr requirements while pregnant and excessive sugar intake.

All of the populations and relationships described in this paper are from different sources. In order To definitely establish these relationships larger more robust studies are needed.

\section{REFERENCES}

[1] Lind J. A treatise on Scurvy. Republished Edinburgh University Press 1953.

[2] Williams RR. Toward the conquest of berberi. Cambridge M.A. Harvard University Press 1961. http://dx.doi.org/10.4159/harvard.9780674593930

[3] Sanhu MS, White IR, Mcpheron K. System review of the prospective cohort studies cohort studies on meat consumption and colorectal list: a meta analytical approach. Cancer Epidemiology Biomarker Prev 2001; 10: 439-446.

[4] American Heart Association 2009. Binge drinking linked with hemorrhagic stroke in Korean men Science Daily 29 June. www.sciencedaily/com/release/2009/06/090625201805htm

[5] United States Department of Agriculture: Assessed at http:www.ars.usda.gov/nutrient data

[6] American academy of Pediatrics. Committee on Nutrition. Pediatric Nutrition Handbook. $5^{\text {th }}$ Ed. Elk Groove, III. American Academy of Pediatrics 2003; pp. 932-933.

[7] Shamberger RJ. Autism rates and the WIC program. J. Am College of Nutrition 2011; 30: 348-353. http://dx.doi.org/10.1080/07315724.2011.10719978

[8] Mukherjee R, Layton M, Yacoub E, Turk J. Autism and autistic traits in people exposed to heavy prenatal alcohol: data from a series of 21 individuals and nested case control study. Advance In mental health and intellectual disabilities 2011; 5: 42-49.

[9] Marchetta CM, Floyd L, Sniezck, McKnight LR. Alcohol and binge drinking among women of childbearing age-United States, 2006-2010. Morbidity and Mortality Weekly Report (MMWR) 2012; 61: 534-538.

[10] Kim YS, Leventhal BL, Koh JL, Fombonne E, Laskete E, Lin EC, Cheon KA, Kim YK, Song DW, Grinkev RR. Prevalence of autism spectrum disorders in a total population sample. Am J Psychiatry: Am J Psychiatry 2011; 168: 904-912. http://dx.doi.org/10.1176/appi.ajp.2011.10101532

[11] Paleodiabetic.com/tag/Jeremy-landen/ Is a Stone Age Diet Healthy for Diabetics.Jeremy-Landen. Posted on April 21, 201

[12] Lonsdale D, Shamberger RJ. Treatment of autism spectrum children with thiamine tetra hydrofurfuryl disulfide. A pilot study. Neuro Endocrinol Lett 2002; 23: 303-308.

[13] Suren P, Roth C, Bresnaham M, Haugen M, Hirtz D, Lie KK, Lipkin WI, Magnus P, Reichborn-Khennerud et al. Association between maternal use of folic acid supplements and risk of Autism spectrum disorders in children. JAMA 2013; 309: 370-377.

[14] James SJ, Melnyl S, Jennigan S, Blossom S, Pavico O Goyner DW. Cellular and mitochondrial Glutathione redox imbalances in lymphoblastoid cells from children with autism. FASEB J 2009; 23: 2374-2383 http://dx.doi.org/10.1096/fi.08-128926

[15] State-Specific Alcohol Consumption for 2010. CDC Centers for Disease Control and Prevention http://www.cdc.gov/ ncbddd/fasd/monitor-table.html

[16] Individuals with Disabilities Act (IDEA-Part B and Part C. $\mathrm{http} / /$ tagnet.public.tagnet.org/pages/712

[17] Robinson JL, Nations L, Suslowitz N, Cuccaro ML, Haines J, Pericak-Vance M. Prevalence rates of autism spectrum disorders among the old order amish. INSAR International Society for Autism Research. https://imsar.Confex.com/imfar/ 2010/webprogram/Paper 7336html.

[18] Cooper DL, Peterick ES, Wright J. The association between binge drinking and birth outcomes: Results from the born in Bradford Endland cohorts. J Epidemiol Community Health doi:10.1136/jec 2012-202303.

[19] Allen LH. B vitamins and breast milk: relative importance of maternal status and effects of infant status and function. Adv $\mathrm{Nu} 2012$; 3: 362-369. http://dx.doi.org/10.3945/an.111.001172

[20] Sakurai T, Furukama M, Asoh M, Kanno T, Kojima T, Yonekubo A. Fat-soluble and water vitamin contents of breast milk from Japanese women. J Nutr Sci Vitaminol (Tokyo) 2005; 51: 239-47. http://dx.doi.org/10.3177/jnsv.51.239

[21] Boylan LM, Hart S, Porter KB, et al. Vitamin B-6 content of breast milk and neonatal behavioral functioning 2002; 102: 1433-1438. 